\title{
Bornean orangutans: primary forest in Danum Valley and rehabilitation program on Orangutan Island
}

\author{
Tetsuro Matsuzawa ${ }^{1,2,3}$
}

Published online: 3 November 2019

๑) Japan Monkey Centre and Springer Japan KK, part of Springer Nature 2019

This is a brief report on my recent trip to Borneo and Peninsular Malaysia in 2019 to see orangutans (Pongo pygmaeus) and other nonhuman primates (Fig. 1). I first went to see wild orangutans in Sabah, Borneo, in 1999. This article aims to look back on my commitment to the Bornean orangutans over the past 20 years.

Geopolitics seems to be not limited to politics, but extends to the academic world as well. European countries have an advantage in the study of nonhuman primates in Africa. They are geographically close to Africa and have long-term relationships with African countries through colonization. North America has an advantage in the study of nonhuman primates in Central and South America. For Japan, it might be valuable to pay more attention to Southeast Asia. Orangutans, great apes of the hominid family, must be a target species of scientific strategy. They live on only two islands, Sumatra and Borneo-in other words, Indonesia and Malaysia. Based on this understanding, I first went to Sabah, Borneo, Malaysia, in the summer of 1999.

\section{Borneo in 1999}

Let me start with reflections on the first visit to Borneo 20 years ago. I was accompanied by five students: Shiho Fujita (a graduate student at that time and now associate professor of Kagoshima University) and Toyomi Matsuno, Aya Saitoh, Misato Hayashi, and Michiko Nagai (all of whom went on to become university professors). We took a flight from Osaka in Japan to Kuala Lumpur, the capital of Malaysia. From there we flew to Kota Kinabalu, the capital of the

Tetsuro Matsuzawa

matsuzawa.tetsuro.8w@kyoto-u.ac.jp

Kyoto University Institute for Advanced Study, Kyoto, Japan

2 Primate Research Institute, Kyoto University, Inuyama, Japan

3 Japan Monkey Centre, Inuyama, Japan state of Sabah, Borneo. Finally, we flew to Sandakan, the second largest city in Sabah.

When the flight was approaching Sandakan, I was surprised to see a huge oil-palm plantation. The word "Borneo" reminds us of words such as "jungle" or "tropical forest". However, the reality was far from my imagination. All natural forests had already disappeared, replaced by oil-palms.

Sandakan was the capital of Northern Borneo under colonization by Britain in the nineteenth century. It was the port for shipping huge amounts of timber cut from Bornean forests for export to the UK and other countries. Sandakan is also a familiar name for me and others of my generation. It is associated with the word "Karayukisan" as the title of a nonfiction novel written by Tomoko Yamazaki in 1972. Karayukisan refers to young Japanese women who were sent to work (mainly as prostitutes) in foreign countries, especially in Southeast Asia, from the end of the nineteenth century until 1945, the end of World War II. It is the story of a lady who was sent to Sandakan and who worked in a brothel. The

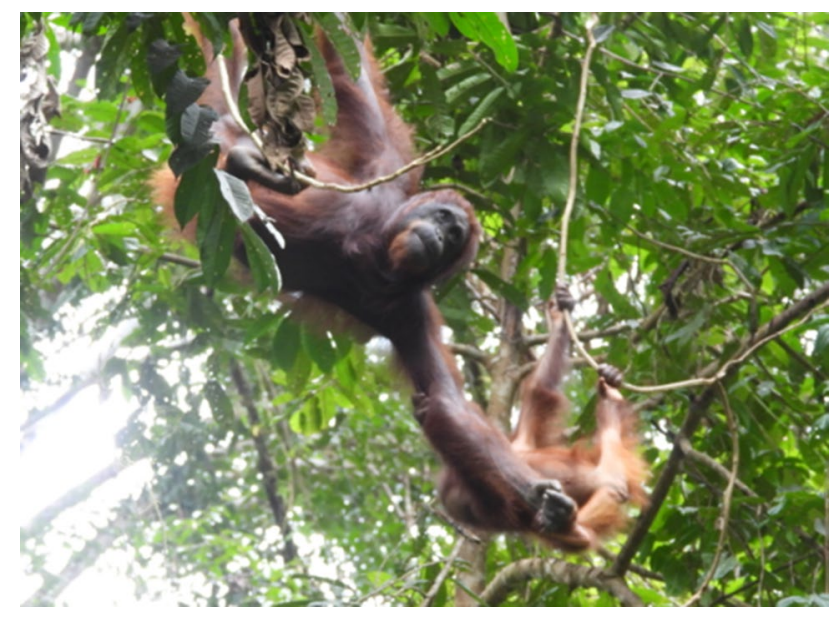

Fig. 1 Orangutans in Danum Valley. The mother Linda and her daughter Lya (photo by Tetsuro Matsuzawa) 
1974 film "Sandakan Hachiban Shokan (Brothel 8)" was directed by Kei Kumai. The actress Kinuyo Tanaka gave an accomplished performance in the film, highly appreciated worldwide, and for which she received the Silver Bear (Best Actress) at the Berlin International Film Festival. Sandakan also reminds us of the Sandakan Death Marches, a series of forced marches in Borneo from Sandakan to Ranau in 1945 that resulted in the deaths of more than 2000 British and Australian prisoners of war held captive by the Empire of Japan during the war. In short, Sandakan is connected to the memories of those sad wartime events. We shall not repeat the evil.

We first went to Sepilok, near Sandakan, where an orangutan sanctuary, the Sepilok Orangutan Rehabilitation Centre (SORC), was located. SORC was established in 1964 and is one of the oldest rehabilitation centers in the world. It has received hundreds of orphaned orangutans from all over Sabah and released them into the neighboring Kabili-Sepilok Forest Reserve $\left(42 \mathrm{~km}^{2}\right)$ and Tabin Wildlife Reserve $\left(1225 \mathrm{~km}^{2}\right)$. We saw orangutans in SORC at close distance. Our first encounter with the orangutans in Sepilok gave us the strong impression of these large animals spending almost their entire lives in the trees.

After that visit of mine, Satoshi Hirata visited Sepilok (and also Danum Valley) twice in successive years. The first visit was with Gaku Ohashi in October-November 1999 to carry out field experiments on tool use in Sepilok (Hirata and Ohashi 2003). The second visit was with Noe Nakashima in 2000. Kuze et al. $(2008,2012)$ reported on the reproductive parameters of female orangutans in Sepilok, comparing them with other sites. Tajima et al. (2018) analyzed the reproductive success of a flanged male and an unflanged male.

Our final destination during the 1999 trip to Borneo was Danum Valley in Sabah. The Primate Research Institute (PRI) of Kyoto University had a long tradition of fieldwork in nonhuman primates in Southeast Asia, founded by the late professor Shunzo Kawamura (1927-2003). Kawamura, one of the students of Kinji Imanishi (1902-1992), the founder of Primatology in Japan, made two major contributions to field studies of Japanese monkeys in the wild. One is the finding of the dominance rule of monkeys, in which young daughters are more dominant to elder siblings (Kawamura 1958). This dominance is shaped through the mothers' help to the younger ones, and it continues even after the death of the mother. The second is the finding of the famous sweet-potato washing in Koshima monkeys as a strong example of cultural behavior of monkeys (Kawamura 1959). Kawamura was also a pioneer in starting fieldwork in nonhuman primates in Southeast Asia, firstly in Sumatra, Indonesia. The tradition was continued by the late Osamu Takenaka, and then by Kunio Watanabe.

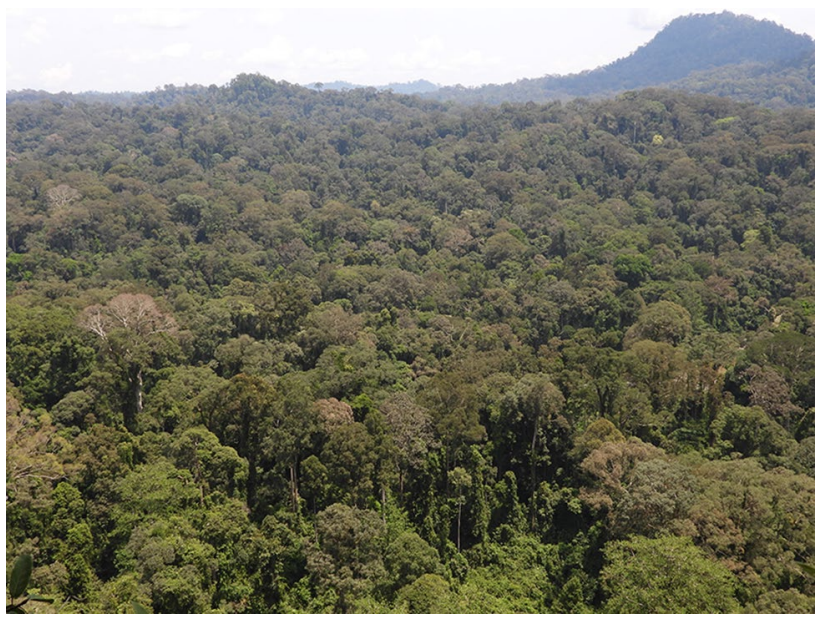

Fig. 2 The primary forest of Danum Valley from the view point (photo by Tetsuro Matsuzawa)

One day I posed a question to my colleague, Prof. Watanabe, who spent his life with the nonhuman primates in Southeast Asia. "Suppose that I can visit only one place in Borneo; where do you recommend me to go?" He immediately said: "Danum Valley". That is the reason I chose Danum Valley as our destination (Fig. 2).

The days we spent in Danum Valley in 1999 remain unforgettable. We enjoyed experiencing the primary forest in Borneo. The dominant tree species are Dipterocarpaceae, 50-70 $\mathrm{m}$ in height. The trees are tall, much taller than those I saw in Africa and the Amazon. There were canopy walks to connect the trees, providing an aerial view of the forests. We enjoyed watching orangutans, gibbons, macaques, and other animals. The night safari gave us the opportunity to observe flying squirrels gliding from one tree to the next. The leeches were everywhere and not so pleasant for the visitors.

\section{Danum Valley, Sabah, Borneo}

Geographic variation in some aspects of chimpanzee behavior has been interpreted as evidence of culture. Similar geographic variation is documented in orangutans as well (van Schaik 2004). The study of orangutans in Borneo was pioneered by John Mackinnon (1974a, b). In Sabah, there is another active research site near the Kinabatangan river (Ancrenaz et al. 2005).

Danum Valley is located in the center of Sabah. The Danum Valley Conservation Area is run by the Sabah Foundation. The area covers $438 \mathrm{~km}^{2}$ of relatively undisturbed lowland dipterocarp forest. The Borneo Rain Forest Lodge (BRFL) was opened for ecotourists. Over the past two decades, based on the memorandum of understanding between the Sabah Foundation and PRI, I have frequently visited the 
area to show its nature to colleagues and students. Among them, I clearly remember Claudia Sousa and Susana Carvalho, my former students from Portugal. I also visited with Shiro Koshima, Yena Kim, Renata Mendonça, and Daisuke Mizuguchi. We climbed to the summit of Mt. Kinabalu, 4095 m (Fig. 3).

Sabah Foundation controls Danum Valley and the other two conservation areas, Maliau Basin and Inbak Canyon. The Wildlife Research Center of Kyoto University was founded in 2008. As the first endeavor of the new center, Prof. Koshima and I organized tours to both places to assess the possibility of studying wild orangutans. According to information from Sabah Foundation, the presence of orangutans was recently confirmed in Inbak Canyon. All three conservation areas have unique features; however, Danum Valley is the richest in fauna and flora.

In Danum Valley you can see seven species of nonhuman primates-orangutans (Pongo pygmaeus morio), Bornean gibbons (Hylobates muelleri), pig-tailed monkeys (Macaca nemestrina), long-tail monkeys (Macaca fascicularis), redleaf monkeys (Presbytis rubicunda), slow loris (Nycticebus spp.), and western tarsiers (Tarsius bancanus) —and other animals including poisonous snakes (Figs. 4, 5, 6, 7, 8). The 2019 expedition consisted of 15 members and a 7-year-old child: four professors, two post-doctoral researchers, one zookeeper, two graduate students, and six undergraduate students. The aim was to introduce Borneo to students who might become field workers in the future.

This time we happened to encounter a huge mass-flowering. It was pointed out by both Dr. Sauwai Yap from Sabah Foundation and Prof. Takakazu Yumoto, the director of PRI and an expert in forest ecology. The mass-flowering in 2019 corresponds to one that happened in 1987 when Prof. Yumoto had been working on canopy ecology in Sarawak,

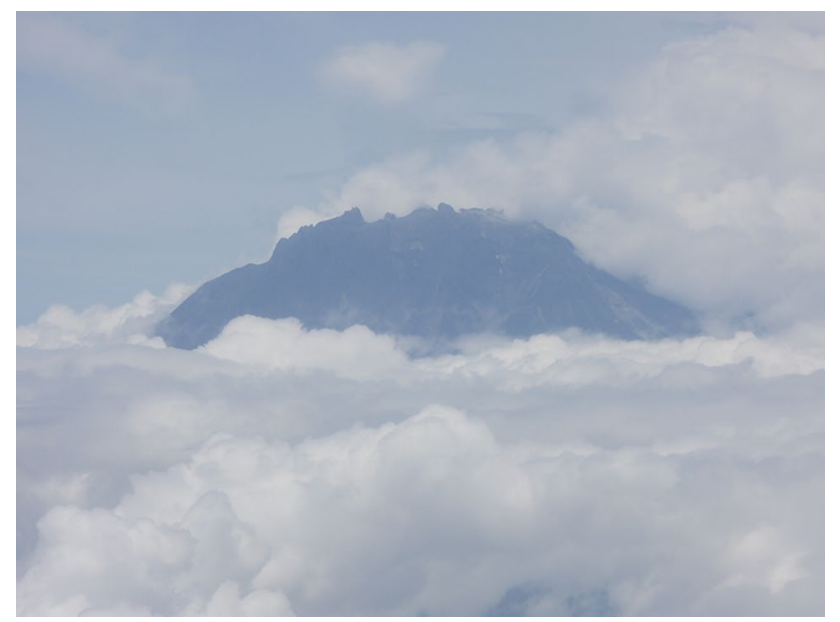

Fig. 3 Mt. Kinabalu (4095 m) viewed from the airplane (photo by Tetsuro Matsuzawa)

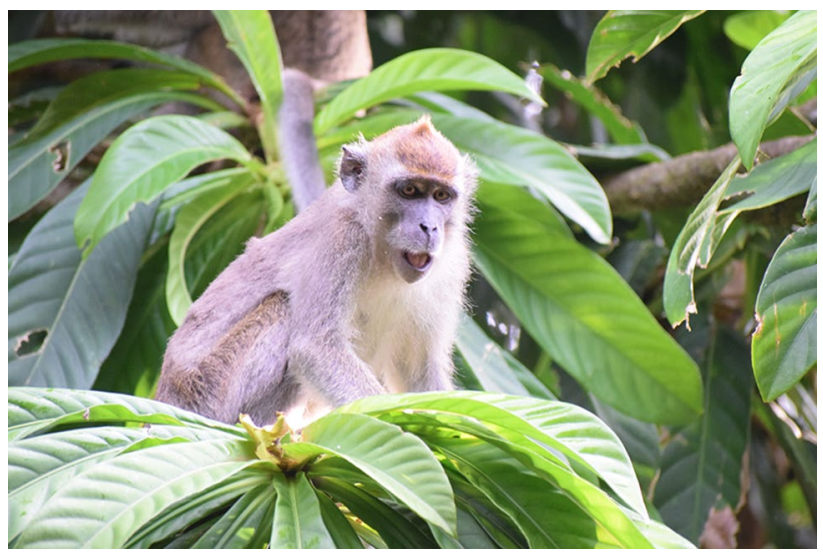

Fig. 4 Long-tail monkey (photo by Tomoe Torii)

a neighboring state to Sabah. He used huge towers to reach the canopy to observe pollination systems of plants joining the mass-flowering event. This new discipline was promoted by Prof. Yumoto with the late Tamiji Inoue (1947-1997), who was killed in a plane crash in Borneo. Prof. Yumoto also visited Danum Valley in the early 1990s and so was very excited to see the mass-flowering on this scale. We witnessed many seeds of dipterocarp falling from the canopy, their two-bladed propellers causing them to twirl as they fell.

We enjoyed watching wild orangutans on four consecutive days of our stay in Danum Valley. It was always a group of three orangutans: a mother named Linda (estimated age 35 years), her daughter Lya ( 5 years old), and a young female named Danum ( 8 years old). They were always together (Fig. 9). Chimpanzees live in groups of many individuals, but in the case of orangutans, when you encounter them in the forest, you usually see only a mother-child pair or a solitary male or female. The inter-birth interval is about 6-9 years (Galdikas 1995; Knott et al. 2009; van Noordwijk and van Schaik 2005; van Schaik 2004). At around this age, young orangutans leave their mother; a young female might

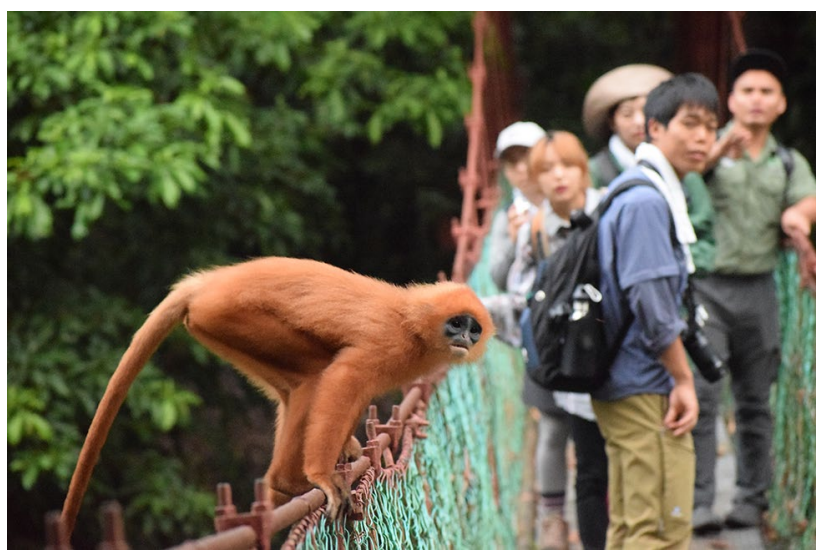

Fig. 5 Red-leaf monkey (photo by Tomoe Torii) 


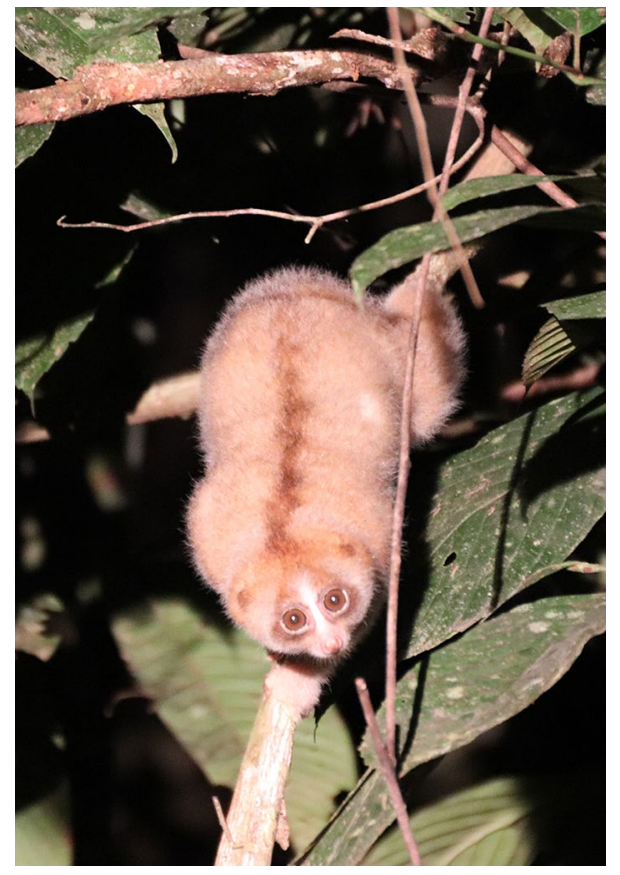

Fig. 6 Slow loris (photo by Takakazu Yumoto)

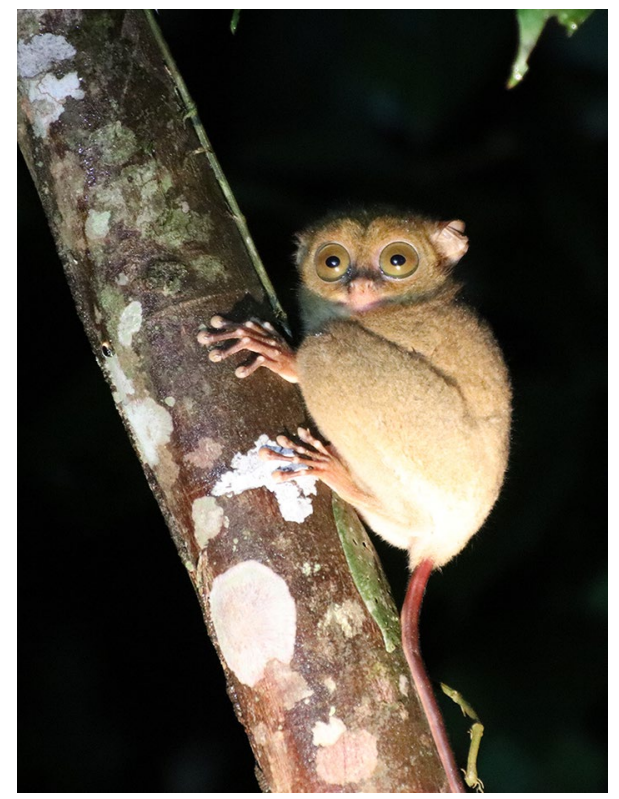

Fig. 7 Tarsier (photo by Takakazu Yumoto)

sometimes join another mother-child pair and thereby start learning how to become a mother.

Danum Valley fieldwork has been led by Tomoko Kanamori and Noko Kuze under the supervision of Shiro Koshima. Later, Tomoyuki Tajima and Renata Mendonça joined, and they have published scientific papers (Kanamori et al. 2010, 2017; Mendonça et al. 2017). The Danum Valley

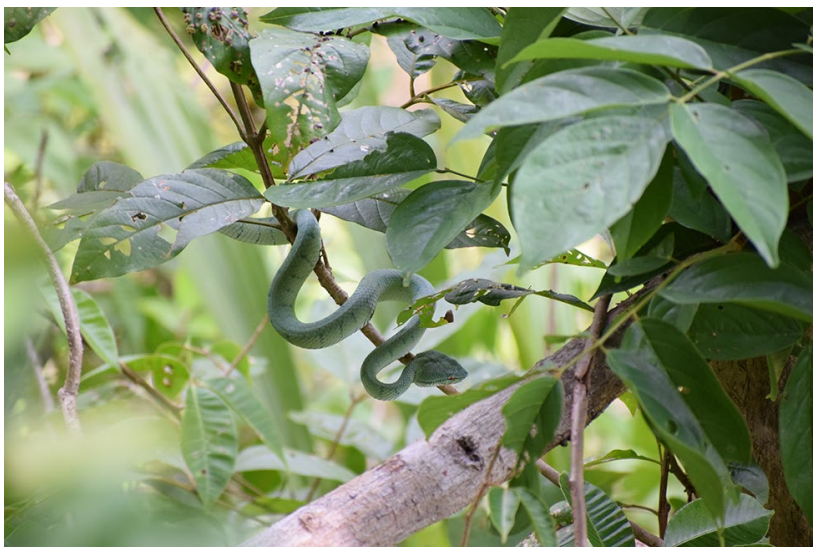

Fig. 8 Bornean keeled pit viper (Tropidolaemus subannulatus) (photo by Tomoe Torii)

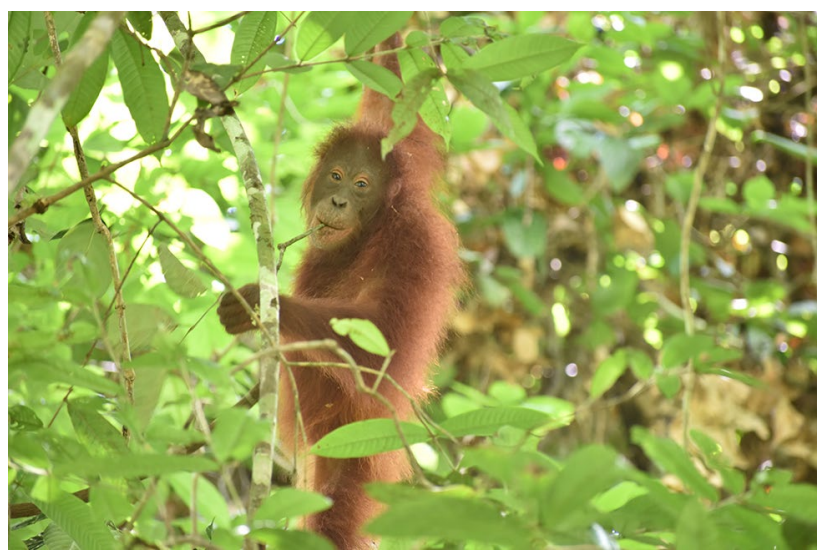

Fig. 9 A young female named Danum 8 years old (photo by Renata Mendonça)

project has continued for 15 years, but it is still a relatively young research site. However, it is a very promising place because of the untouched primary forest left in the heart of Borneo.

\section{Orangutan Island and the BJ island project}

In 2009, 10 years after the first visit to Borneo, we built a research station in Danum Valley as part of the collaboration between the Wildlife Research Center of Kyoto University and the Sabah Foundation. This provided the base for a long-term study of orangutans there (Fig. 10). On the way to the opening ceremony for the station, we visited Mr. Masahiko Horie, Japanese ambassador to Malaysia. He asked me to give advice to the rehabilitation program run by the Malaysian people. EMKAY Foundation, owned by Mr. Tan Sri Mustapha Kamal, had just started a sanctuary program for orangutans on Orangutan Island (OUI) in Bukit 


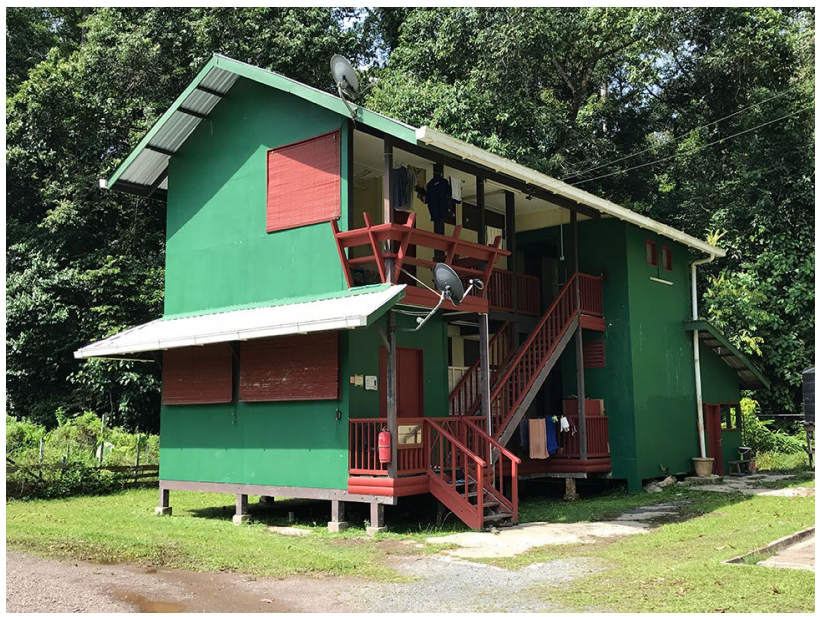

Fig. 10 Field station in Danum Valley (photo by Tetsuro Matsuzawa)

Merah, in the state of Perak, near Penang. The program aims to increase the number of orangutans and return them to the forests. The first orangutans of OUI came from Sarawak, and some others came from zoos.

The Director of OUI was Dr. Sabapathy Dharmalingam. His major was veterinary medicine. When female orangutans gave birth, the infants were separated from the mother (when the infants experienced some health problems) and subsequently reared by humans. Thus, the females repeatedly become pregnant. The number of individuals increases in this situation, but the young orangutans may develop problems as a consequence of maternal deprivation. The infants had no opportunity to learn from their mothers. For young orangutans raised by human caretakers, survival in the natural forests of Borneo must be difficult. They must progress step by step toward coping with real-life challenges if they are to survive independently. In collaboration with Dr. Dharmalingam and my Malaysian counterpart, Prof. Masshor Mansor of Malaysia Science University (USM), we created a new program to have orangutans living on an island to experience free-ranging conditions in the forest.

After further interaction in Malaysia and in Japan, we decided to release three orangutans on an island called BJ island (Fig. 11), not far from OUI. The BJ island project was formally launched on February 16, 2011. The three orangutans released were Ah Ling, a 14-year-old young adult male brought from Sarawak; Nicky, a 19-year-old adult female brought from Sarawak; and Sonia, an 8-year-old juvenile female. The three orangutans spontaneously made sleeping platforms in the trees from the very first night. Misato Hayashi was appointed as principal investigator on the project, and she has continued monitoring the thee orangutans since their release (Hayashi et al. 2018).

Nicky was actually pregnant when she went to BJ island. She gave birth to a son, named William, on March 21, 2011,

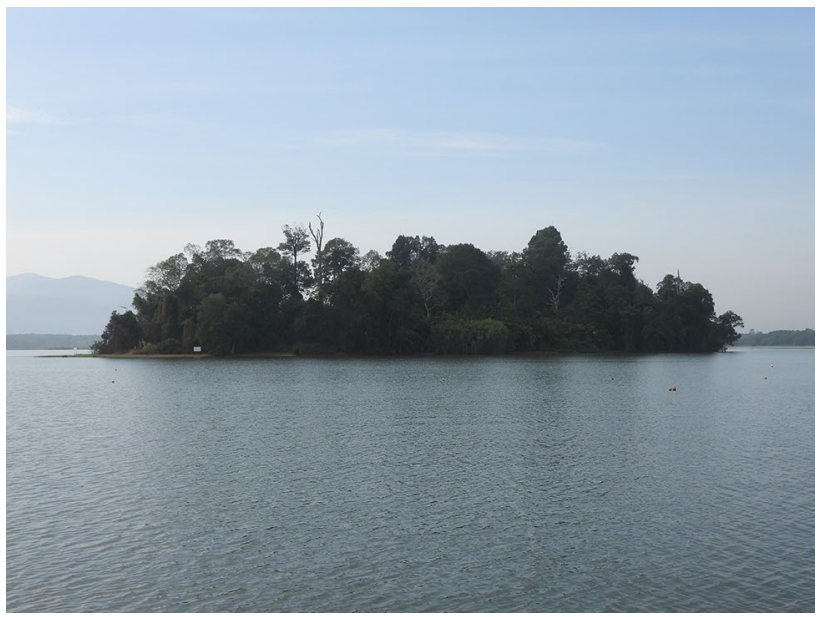

Fig. 11 BJ island (photo by Tetsuro Matsuzawa)

around the time of the Royal wedding of Prince William and Miss Catherine Middleton in the UK. Malaysia has a longterm connection to the UK, so the people of OUI wanted to give the name "William" to the baby boy orangutan. It was satisfying to see the infant living in the forest and learning many things from the mother (Fig. 12). Unfortunately, William passed away at the age of 2 years 4 months because of a respiratory disease. However, Nicky got pregnant again; the father must be Ah Ling. Nicky gave the birth to another son, named Chacha, on September 15, 2014. This proved that $\mathrm{BJ}$ island was suitable for orangutan reproduction as well.

BJ island covers 5.6 ha. USM personnel carried out a vegetation survey before the release. The island was covered by 635 trees from 35 families, 70 genera, and 102 species. Some food was supplied by caretakers because the island was not large enough; however, the three orangutans spontaneously started utilizing 14 species as food. They spent more time up in the trees than on the ground. Time spent moving

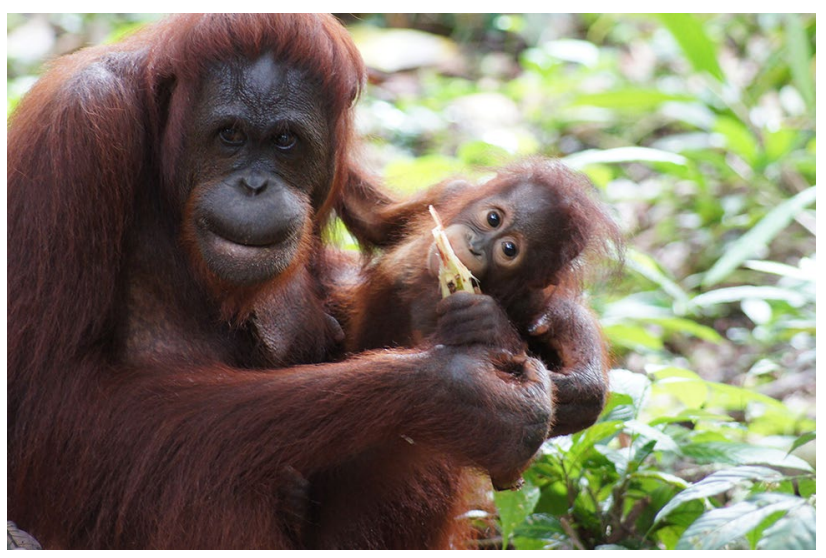

Fig. 12 Mother Nicky and her son William on BJ island (photo by Misato Hayashi) 
increased whereas time spent resting decreased on $\mathrm{BJ}$ island compared to OUI. The relocation to BJ island was shown to be a good step between captivity and the forest.

The $\mathrm{BJ}$ island project is paused at this moment, as financial support for the new endeavor did not continue. Furthermore, OUI suffered from last year's serious fire. We landed on BJ island this time, and saw that it had become jungle, as it was before. However, there is hope. One individual born on OUI was successfully relocated to Sarawak forest in 2018. We hope that the attempt continues, although success may vary.

\section{What I learned from Borneo}

Japan has made a unique contribution to the study of nonhuman primates in Southeast Asia (Matsuzawa 2015). PRI has been hosting training programs for young primatologists from Asian countries, such as the ASIAN-HOPE program (https://www.pri.kyoto-u.ac.jp/sections/asian-hope/index .html). This has provided opportunities for young scholars from Indonesia, Malaysia, Vietnam, Laos, Cambodia, Myanmar, Thailand, and India as well. Scientists from those habitat countries may promote fieldwork on orangutans, gibbons, proboscis monkeys, macaques, and other primates.

Many scholars have focused on African chimpanzees, bonobos, and gorillas to better understand human evolution. Fewer have specialized in orangutans and gibbons in Southeast Asia. However, the arboreal lifestyles of orangutans and gibbons are of such interest for looking at questions from a different perspective. Consider hominid evolution: From common ancestors, African great apes (chimpanzees, bonobos, and gorillas) preferred to stay in the forest. Their forest life is half arboreal and half terrestrial. Humans preferred to venture from the forest out into the savanna. Although of similar body size, orangutans really went in the opposite direction: a fully arboreal life, mostly keeping away from the ground. That might be another story of human evolution. "Oran" means "human" and "Utan" means "forest" in Malay language. Comparisons of the two extremes, humans and orangutans, may bring unique features of human evolution into a clearer light.

Society is important in understanding the difference between humans and orangutans. Orangutans live a largely solitary life, although there is a long and intense relationship between mothers and their offspring. The orangutan triad of Linda-Lya-Danum clearly illustrates the education-master apprenticeship for observational learning of how to raise a child. A young juvenile female sometimes moves with a mother-infant pair and learns how to mother. I have also witnessed a case of sexual intercourse between a juvenile male and a multiparous female. In Danum Valley, females seem to stay in relatively small home ranges, while males move around a wider area. So far, the field study in Danum Valley has been limited to looking around the area on 1-day treks from the field station. This is like trying to observe the sky through a tiny hole from a fixed point. It is important to continue the observation, but we need more efforts to see orangutans in the wider area. This would provide answers to questions about the life history of male orangutans: when and how they become adults.

Studies of material intelligence such as tool use and manufacture have largely focused on chimpanzees. Although orangutans show the same level of intelligence in captivity (Russon and Galdikas 1993), and also use tools in the wild (van Schaik et al. 2003), it is not fair to compare their tool behavior in the wild with that of chimpanzees, because orangutans' four hands are busy supporting their body in the trees. Another important aspect of intelligence might concern utilization of three-dimensional space in the forest. Humans mostly remain on the ground, which means that they are especially active in two-dimensional space. By contrast, orangutans in the trees are always confronted with the problem of how to move from one place to the next. This must be very demanding intellectually. Orangutans are very large and heavy, and not all trees can support their body weight. To reach the fruits in one tree, you must solve detour problems; a quick look and quick decision will not work. You need to take time to understand the structural properties and configurations of the tree trunk and the branches to move from one place to the next. Satoshi Hirata proposed the "detour intelligence hypothesis" in orangutans, taking into account the unique niche of orangutans in the forest (Hirata 2001).

I have mentioned unique aspects of society and intelligence in orangutans. The most important thing for studying orangutans is continuity. How do we continue fieldwork? We have to raise and train researchers in Japan and other countries, including Malaysia. We need trackers who know the fauna and flora, culture, and history of the research site. For that purpose, we need to develop the logistics and continue giving necessary financial, material, and mental support. The trip to Borneo in 2019 gave me the opportunity to look back on earlier efforts, and to recognize what was lacking in the past and what is necessary for the future.

Acknowledgements I am grateful to the following organizations in Malaysia: Sabah Foundation, Sabah Wildlife Department, University of Sabah in Malaysia, Malaysia Science University. I also thank the following individuals for their help and encouragement at various stages of the study in Borneo: Tan Sri Mustapha Kamal, Masshor Mansor, Shahrul Anuar Mohd Sah, Sabapathy Dharmalingam, Daniel Baskaran, Henry Bernard, Titol Peter Malim, Waidi Sinum, Sauwai Yap, Hisashi Matsubayashi, and Masahiko Horie. The trip to Borneo was advised and facilitated by Kunio Watanabe, Tomoko Kanamori, Noko Kuze, and Ikki Matsuda. The trips to Borneo have been supported by MEXTJSPS grants listed at the end. The 2019 trip was financially supported by the Kyoto University Presidential Program: Prof. Juichi Yamagiwa 
is the president. Special thanks are due to the members of the 2019 trip. We shared a pleasant time in Malaysia: Shiro Kohshima, Takakazu Yumoto, Misato Hayashi, Saki Hayashi, Tomoko Kanamori, Renata Mendonça, Takashi Funahashi, Mikuho Yokoyama, Tomoe Torii, Toshiki Minami, Kaede Yokosaka, Risa Fumimoto, Tamayo Tomoda, Rina Yukino, and Tomoharu Ikeda. Special thanks are due to the personnel who helped us on-site: Tomoko Kanamori, Tomoyuki Tajima, and Eddy Boy. Without their kind arrangements, it would have been impossible to have a pleasant time. Financial support for preparing the manuscript came from MEXT-JSPS Grants \#24000001, \#16H06283; the Japan Society for the Promotion of Science (JSPS) Core-to-core Program CCSN, and the Leading Graduate Program of Primatology and Wildlife Science (U04) to the author. I also thank Prof. James Anderson of Kyoto University for help with editing the English text.

\section{References}

Ancrenaz M, Gimenez O, Ambu L, Ancrenaz K, Andau P, Goossens B, Payne J, Sawang A, Tuuga A, Lackman-Ancrenaz I (2005) Aerial surveys give new estimates for orangutans in Sabah, Malaysia. PLoS Biol 3:e3

Galdikas BMF (1995) Reflections of Eden. Little Brown, Boston

Hayashi M, Kawakami F, Roslan R, Hapiszudin NM, Dharmalingam S (2018) Behavioral studies and veterinary management of orangutans at Bukit Merah Orang Utan Island, Perak, Malaysia. Primates 59:135-144

Hirata S (2001) Intelligence viewed from arboreal travel: tool use of primates, detour hypothesis, and social intelligence. Ecosophia 8:98-107 (in Japanese)

Hirata S, Ohashi G (2003) An experimental study of tool use in orangutans. Primate Res 19:87-95 (in Japanese with English summary)

Kanamori T, Kuze N, Bernard H, Malim P, Kohshima S (2010) Feeding ecology of Bornean orangutans (Pongo pygmaeus morio) in Danum Valley, Sabah, Malaysia: a 3-year record including two mast fruitings. Am J Primatol 72:820-840

Kanamori T, Kuze N, Bernard H, Malim TP, Koshima S (2017) Fluctuations of population density in Bornean orangutans (Pongo pygmaeus morio) related to fruit availability in the Danum Valley, Sabah, Malaysia: a 10-year record including two mast fruitings and three other peak fruitings. Primates 58:225-235

Kawamura S (1958) Mother-daughters dominance structure in Minoo B troop: a study of the dominance rank in Japanese monkeys. Primates 1:149-156 (in Japanese with English summary)
Kawamura S (1959) The process of sub-culture propagation among Japanese macaques. Primates 2:43-60

Knott CD, Emery Thompson M, Wich SA (2009) The ecology of female reproduction in wild orangutans. In: Wich SA, Utami Atmoko SS, Mitra Setia T, van Schaik CP (eds) Orangutans: geographic variation in behavioral ecology and conservation. Oxford University Press, New York, pp 171-189

Kuze N, Sipangkui S, Malim T, Bernard H, Ambu L, Kohshima S (2008) Reproductive parameters over a 37-year period of free ranging female Borneo orangutans at Sepilok Orangutan Rehabilitation Centre. Primates 49:126-134

Kuze N, Dellator D, Banes GL, Pratje P, Tajima T, Russon AE (2012) Factors affecting reproduction in rehabilitant female orangutans: young age at first birth and short inter-birth interval. Primates $53: 181-192$

Mackinnon J (1974a) The behaviour and ecology of wild orang-utans (Pongo pygmaeus). Anim Behav 22:3-74

Mackinnon J (1974b) In search of the red ape. Collins, London

Matsuzawa T (2015) Sumatran orangutans and the World War II. Primates 56:207-209

Mendonça RS, Kanamori T, Kuze N, Hayashi M, Bernard H, Matsuzawa T (2017) Development and behavior of wild infant-juvenile East Bornean orangutans (Pongo pygmaeus morio) in Danum Valley. Primates 58:211-224

Russon A, Galdikas B (1993) Imitation in free-ranging rehabilitant orangutans (Pongo pygmaeus). J Comp Psychol 107:147-161

Tajima T, Malim TP, Inoue E (2018) Reproductive success of two male morphs in a free-ranging population of Bornean orangutans. Primates 59:127-133

van Noordwijk MA, van Schaik CP (2005) Development of ecological competence in Sumatran orangutans. Am J Phys Anthropol 127:79-94. https://doi.org/10.1002/ajpa.10426

van Schaik CP (2004) Among orangutans: red apes and the rise of human culture. Harvard University Press, Cambridge

van Schaik CP, Ancrenaz M, Borgen G, Galdikas B, Knott CD, Singleton I, Suzuki A, Utami SS, Merrill M (2003) Orangutan cultures and the evolution of material culture. Science 299:102-105

Publisher's Note Springer Nature remains neutral with regard to jurisdictional claims in published maps and institutional affiliations. 